\title{
Time Course of Cerebral Perfusion Changes in Children with Migraine with Aura Mimicking Stroke
}

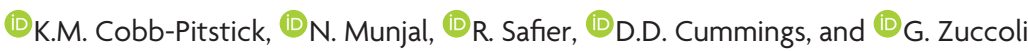

\begin{abstract}
SUMMARY: Hemiplegic migraine is a common cause of acute brain attack in pediatrics. MR imaging sequences useful in differentiating hemiplegic migraine from other entities include arterial spin-labeling, SWI, MRA, and DWI. There has been limited exploration on the simultaneous use of these sequences in pediatrics. We present 12 pediatric patients with acute hemiplegic migraine or migraine with aura who underwent MR imaging within 12 hours of symptom onset. Quantitative and qualitative analyses were performed on arterial spinlabeling; and qualitative analysis, on SWI and MRA sequences. All 12 patients had normal DWI and abnormal arterial spin-labeling findings. Furthermore, we observed a more rapid transition from hypoperfusion to rebound hyperperfusion in 3 patients compared with prior reports. These findings support the use of multimodal MR imaging to distinguish migraine with aura from stroke and the simultaneous use of these MR imaging sequences to improve understanding of perfusion changes during migraine with aura.
\end{abstract}

ABBREVIATIONS: $\mathrm{ASL}=$ arterial spin-labeling; $\mathrm{rCBF}=$ relative $\mathrm{CBF}$

$\mathrm{H}$ emiplegic migraine is one of the most common causes of acute brain attack (defined as acute onset of focal neurologic symptoms) seen in the pediatric emergency department, accounting for approximately one-third of pediatric brain attacks, and it can be difficult to differentiate from arterial ischemic stroke or TIA on initial presentation. ${ }^{1,2}$ Hemiplegic migraine is defined according to the International Classification of Headache Disorders, 3 rd edition ${ }^{2}$ criteria by the presence of an aura characterized by fully reversible motor and visual, sensory, and/or speech/language symptoms and at least 2 of the following characteristics: 1 ) At least 1 aura symptom spreads gradually for $\geq 5$ minutes and/or $\geq 2$ symptoms occur in succession; 2 ) each individual aura symptom lasts 5-60 minutes, and motor symptoms generally last $<72$ hours (but may persist for weeks); 3) at least 1 aura symptom is unilateral; and 4) the aura is accompanied by or followed within 60 minutes by headache. ${ }^{3}$ The pathophysiology of migraine and migraine with aura has not been determined, but leading hypotheses suggest a cortical spreading depression and subsequent local hypoperfusion as a trigger. ${ }^{4,5}$

Received January 19, 2018; accepted after revision April 11.

From the Department of Pediatrics (K.M.C.-P., N.M., R.S., D.D.C.), Division of Child Neurology, and Division of Pediatric Neuroradiology (G.Z.), Children's Hospital of Pittsburgh of University of Pittsburgh Medical Center, Pittsburgh, Pennsylvania. Katherine M. Cobb-Pitstick and Neil Munjal contributed equally to this work.

Please address correspondence to Giulio Zuccoli, MD, Department of Radiology, Children's Hospital of Philadelphia, 3401 Civic Center Blvd, Wood Building 2115, Philadelphia, PA 19104; e-mail: giulio.zuccoli@gmail.com; zuccolig@email.chop.edu

http://dx.doi.org/10.3174/ajnr.A5693
MR imaging is becoming increasingly available for rapid assessment of pediatric brain attacks, even in patients who would previously have required sedation. Given the prevalence of migraine in the differential diagnosis of pediatric brain attack, reliable neuroimaging features differentiating migraine from acute ischemic stroke or TIA represent a valuable tool in guiding the appropriate level of initial work-up and management. MR imaging sequences that can be useful in differentiating hemiplegic migraine from other entities include arterial spin-labeling (ASL), SWI, and MRA. ASL implements a radiofrequency pulse that labels arterial blood water below the ROI and then captures the resulting signal as the labeled blood enters the ROI. These data are subtracted from the corresponding data obtained from a control sequence that does not label arterial blood water. ${ }^{6}$ The degree of signal alteration correlates with the extent of arterial perfusion. SWI uses a gradient-echo pulse sequence to produce contrast and is blood oxygen level-dependent. The name derives from the use of the phase map of susceptibility difference in adjacent tissue and is the basis for blood oxygen level-dependent fMRI imaging. MRA provides a noninvasive, non-contrast-enhanced technique to image blood vessels. It can be viewed in the original 2D source or reconstructed into a 3D vessel image. Research has been published on the use of various MR imaging sequences to characterize the time course of perfusion changes in migraine, but there has previously been limited exploration of the simultaneous use of ASL, SWI, and MRA, particularly in pediatrics. ${ }^{7-14}$ Our objective was to explore the use of multiple, noninvasive MR imaging sequences to better understand the relationship between clinical 


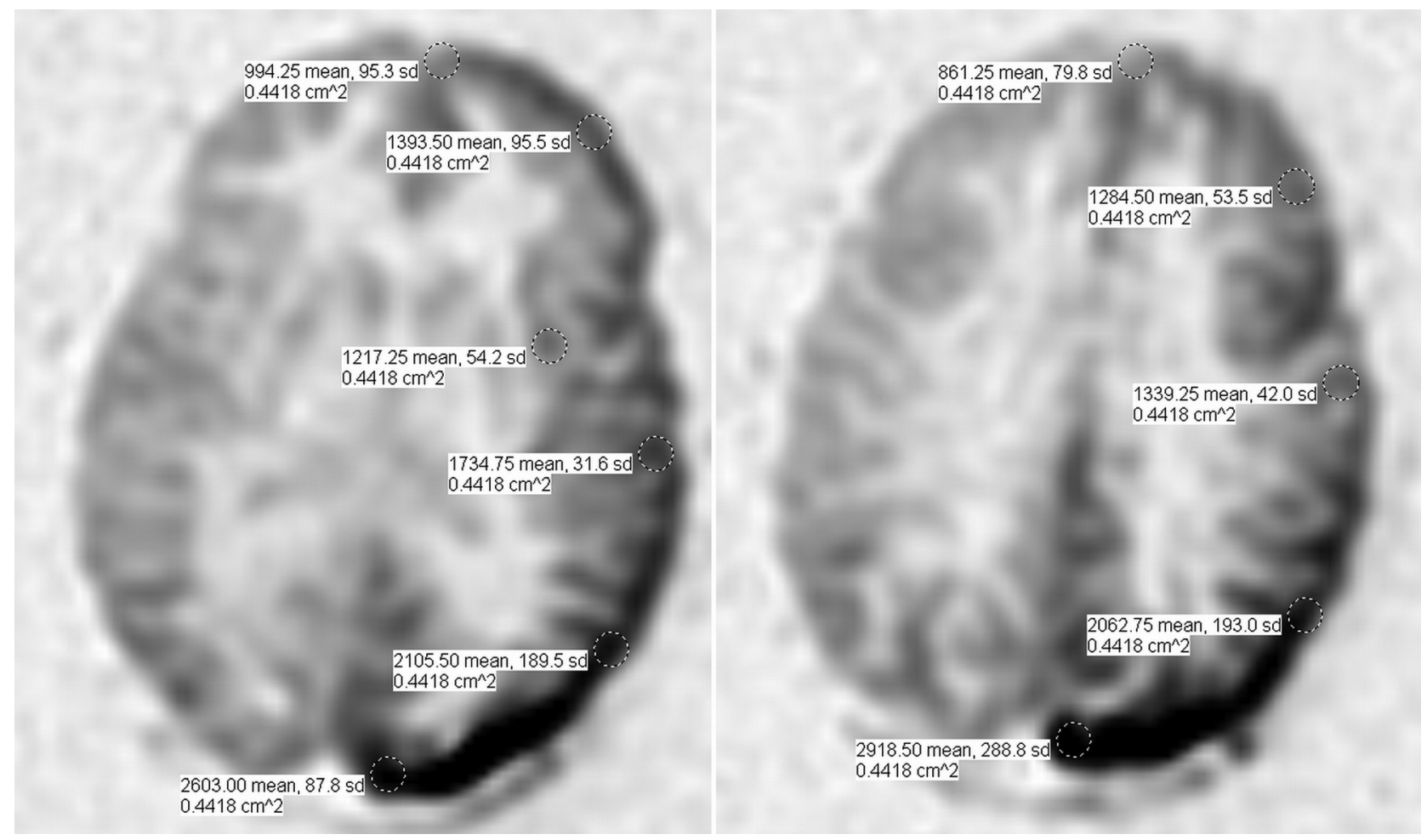

FIG 1. Modified ASPECTS system with 11 ROls centered in the cortex at 2 contiguous levels on ASL imaging.

and MR imaging findings in pediatric hemiplegic migraine and migraine with aura.

\section{Case Series}

This is a case series of children with migraine with focal neurologic symptoms and unilateral MR imaging findings who presented to the Children's Hospital of Pittsburgh emergency department. All cases were collected retrospectively under an institutional review board-approved study. Information was obtained from each patient's medical record and included clinical symptoms, family history, timing of MR imaging from the onset of symptoms, and headache severity. These data came from emergency department documentation and neurology consult notes. Patients were included in the study if they had undergone a stroke-protocol brain MR imaging within 12 hours of neurologic symptom onset, which included DWI, ASL, SWI, 3D-TOF MRA, and FLAIR imaging. Patients were excluded if symptoms were resolved at presentation, MR imaging was not performed before patient discharge from the emergency department or hospital, or MR imaging was performed $>12$ hours after symptom onset.

Qualitative MR imaging findings were identified for ASL, SWI, and MRA sequences. The images were obtained using a $1.5 \mathrm{~T}$ scanner (Signa; GE Healthcare, Milwaukee, Wisconsin) with a 32-channel head coil. The technical factors used for ASL, SWI, and 3D-TOF MRA were the following: pseudocontinuous ASLreceiver bandwidth $=62.50$, imaging mode $=3 \mathrm{D}$, acceleration factor $=1.00, \mathrm{FOV}=24.0$, slice thickness $=4.0$, frequency $=512$, phase $=8$, frequency direction $=$ anteroposterior, NEX $=3.00$, autoshim $=$ auto, phase correction $=$ none; $3 \mathrm{D}$ SWI-flip angle, $15^{\circ}, \mathrm{TE}=50.0 \mathrm{~ms}, \mathrm{TR}=78.3 \mathrm{~ms}$, receiver bandwidth $=41.67$, filter choice $=$ none, $\mathrm{FOV}=20.0$, slice thickness $=3.0$, slab $=32$, overlap $=0$, frequency $=288$, phase $=224$, frequency direction $=$ anteroposterior, phase FOV $=1.00$, autoshim $=$ auto, phase correction $=$ none; $3 \mathrm{D}-\mathrm{TOF}-$ flip angle $=20^{\circ}, \mathrm{TE}=\mathrm{min}-$ imum, number of echoes $=1, \mathrm{TR}=23.0 \mathrm{~ms}$, receiver bandwidth $=31.25$, filter choice $=$ none, imaging mode $=3 \mathrm{D}$ pulse sequence; TOF echo-spoiled gradient-echo-acceleration factor $=1.00, \mathrm{FOV}=16$, slice thickness $=1.4$, localizer per slab $=$ 24 , overlap locations $=6$, frequency $=320$, phase $=224$, frequency direction $=$ anteroposterior, $\mathrm{FOV}=1.00$.

Images were independently reviewed by 1 senior pediatric neuroradiologist and a pediatric neurologist blinded to the affected side for changes in brain perfusion on ASL, signal intensity of the cortical veins on SWI, and caliber of the intracranial arteries on 3D-TOF MRA, respectively. The final interpretation was reached by consensus in cases of disagreement between the readers. Eleven standard ROIs were selected in 2 axial planes on ASL sequences based on standard ASPECTS regions. ${ }^{15}$

Relative CBF (rCBV) values were calculated for standard 0.44$\mathrm{cm}^{2}$ circular regions at each ROI by using a PACS, and the affected side was subtracted from the nonaffected side. These rCBF differences were averaged to produce a net hemispheric blood flow difference. Of note, ROIs on the ASPECTS system are predominantly distributed in MCA territory. In this study, our extreme anterior and posterior ROIs were relocated as demonstrated in Fig 1 to provide anterior cerebral artery and posterior cerebral artery coverage. Quantitative statistical analysis was performed using the R statistical package (https://www.r-project.org).

Twelve patients met the inclusion criteria for this series. There were 6 female patients (50\%) and 6 male patients (50\%). Patients ranged from 9 to 16 years of age. Symptoms were localized to 


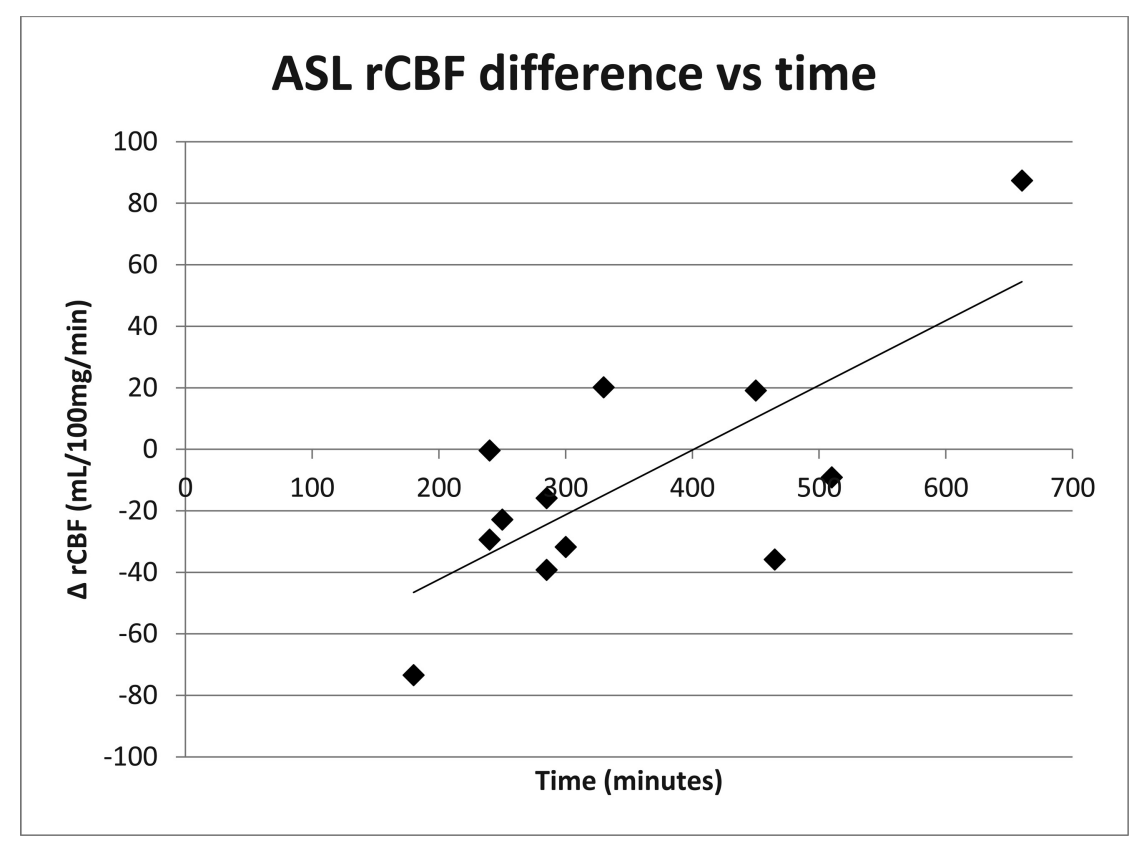

FIG 2. Time course of the brain perfusion changes in migraine with aura from symptom onset.
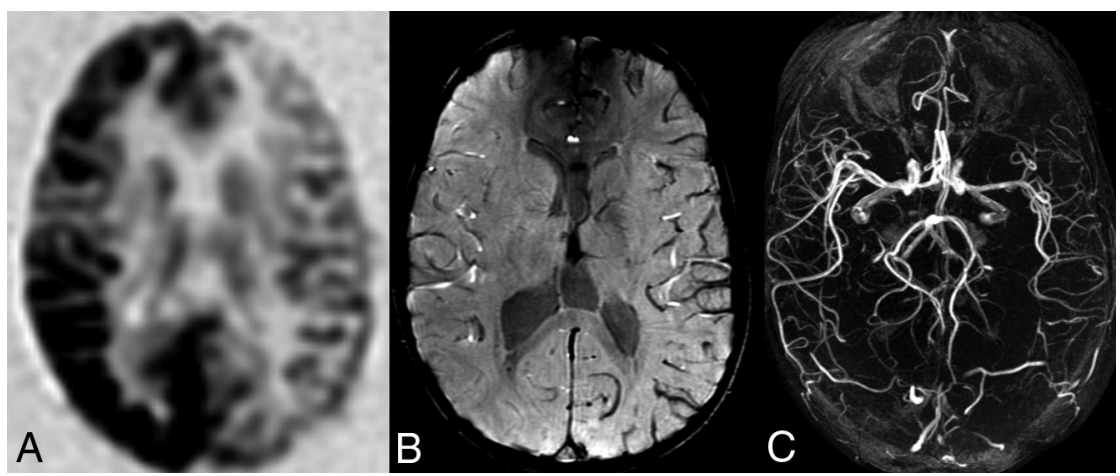

FIG 3. Patient 1. ASL demonstrates left hemispheric hypoperfusion $(A)$. Note hypo-oxygenated cortical veins on SWI along the left cerebral hemisphere (B). MRA demonstrates thinning of the distal branches of the left MCA and posterior cerebral artery $(C)$.

the right hemisphere in 4 patients and the left hemisphere in 8 patients. Brain MR imaging was performed between 3 and 11 hours from neurologic symptom onset. The median time of MR imaging following symptom onset was 4.9 hours. All patients demonstrated a difference in cerebral perfusion on ASL sequences compared with the unaffected hemisphere. Eight patients demonstrated a relative decrease in perfusion on ASL sequences. Of these patients, the 7 who had SWI and MRA available demonstrated a corresponding decreased signal and decreased caliber of vessels, respectively. Patient 12 did not have SWI or MRA sequences available for review. Patient 10 had normal SWI and MRA findings, and the ASL sequence demonstrated a signal difference of -0.4 . Three patients had a relative increase in perfusion on ASL sequences. Patient 2 had normal SWI findings with an increased caliber of vessels on MRA. Patient 7 had normal SWI findings with a decreased vessel caliber on MRA. Patient 11 had normal SWI and MRA findings. DWI sequences for all patients were negative for restricted diffusion. The rCBF and time of MR imaging from symptom onset are shown in Fig 2. An example of the imaging findings during migraine with aura on multimodal MRI sequences for patient 1 is shown in Fig 3 . The available demographic, clinical, time to MR imaging from symptom onset, and imaging features including relative cerebral blood flow are described in the Table. There was a significant positive relationship between the $\mathrm{rCBF}$ and time to MR imaging from symptom onset, $r(10)=0.74, P<.05$.

\section{DISCUSSION}

Information has been published on the use of various MR imaging sequences to characterize the time course of perfusion changes in migraine. Several case series on the use of noncontrast MR imaging in migraine have demonstrated prominent cerebral vasculature in the affected hemisphere on SWI sequences in the evaluation of the acute phase of migraine..$^{7-10}$ ASL is an increasingly more popular, available, and noninvasive MR imaging perfusion sequence that can provide insight into the pathophysiology of pediatric brain attacks, including pediatric migraine and stroke. ${ }^{10}$ The presence of decreased relative cerebral perfusion on ASL in a DWI sequence with normal findings supports the simultaneous use of these sequences as the best MR imaging diagnostic tool for differentiating hemiplegic migraine from stroke. This combination was noted in 8 patients in this case series. ASL asymmetries in stroke or TIA would be localized to a specific vascular territory, while in our patients, migraine-with-aura asymmetries are seen throughout the affected hemisphere in multiple vascular territories. Moreover, lack of restricted diffusion while the patient is clinically symptomatic suggests migraine with aura over stroke.

Many studies have suggested the implementation of ASL in the evaluation of children presenting with focal neurologic symptoms, each with the finding of initial hypoperfusion followed by delayed hyperperfusion. ${ }^{11-14}$ Boulouis et $\mathrm{al}^{11}$ published a case series exploring the use of the ASL MR imaging sequence in children, which demonstrated decreased regional cerebral blood flow when obtained $<14$ hours after symptom onset and increased regional cerebral blood flow when obtained $>17$ hours after symptom onset. Lehman et a ${ }^{12}$ demonstrated decreased pulsed ASL signal in 11 children who all had increased prominence of cortical or medullary veins on SWI, suggesting increased venous deoxyhemoglobin. In the Lehman et al study, ASL hypoperfusion persisted as long as 16.25 hours. Iizaku et $\mathrm{al}^{13}$ also evaluated the changes in perfusion in 3 adult patients with focal neurologic symptoms and migraine whose MR imaging demonstrated initial hypoperfusion and delayed hyperperfusion on ASL $>18-24$ 


\begin{tabular}{|c|c|c|c|c|c|c|c|}
\hline $\begin{array}{l}\text { Patient } \\
\text { No. }\end{array}$ & $\begin{array}{l}\text { Age } \\
\text { (yr) }\end{array}$ & Sex & Symptoms & $\begin{array}{c}\text { Time of MRI from } \\
\text { Symptom Onset (min) }\end{array}$ & SWI & MRA & $\begin{array}{c}\text { ASL rCBF } \\
(\mathrm{mL} / 100 \mathrm{mg} / \mathrm{min})\end{array}$ \\
\hline 1 & 10 & $\mathrm{~F}$ & R paresis & 180 & $\downarrow$ & $\downarrow^{a}$ & -73 \\
\hline 2 & 13 & $\mathrm{~F}$ & R paresis/anesthesia & 660 & Normal & $\uparrow$ & 87 \\
\hline 3 & 13 & M & L paresis/anesthesia & 285 & $\downarrow$ & $\downarrow^{a}$ & -15.8 \\
\hline 4 & 12 & $\mathrm{~F}$ & $\mathrm{~L}$ anesthesia & 240 & $\downarrow$ & $\downarrow$ & -29.3 \\
\hline 5 & 14 & $M$ & $\mathrm{R}$ paresis & 510 & $\downarrow$ & $\downarrow$ & -9.0 \\
\hline 6 & 15 & $\mathrm{~F}$ & $\mathrm{R}$ paresis/anesthesia & 300 & $\downarrow$ & $\downarrow b$ & -31.7 \\
\hline 7 & 13 & M & $\mathrm{R}$ paresis & 330 & Normal & $\downarrow$ & 20.2 \\
\hline 8 & 9 & $M$ & $\mathrm{~L}$ anesthesia & 465 & $\downarrow$ & $\downarrow$ & -50.1 \\
\hline 9 & 9 & M & $\mathrm{L}$ paresis & 285 & $\downarrow$ & $\downarrow^{a}$ & -47.1 \\
\hline 10 & 15 & $\mathrm{~F}$ & R paresis/anesthesia & 240 & Normal & Normal & -0.4 \\
\hline 11 & 16 & $M$ & R paresis/anesthesia & 450 & Normal & Normal & 19.2 \\
\hline 12 & 12 & $\mathrm{~F}$ & R paresis/anesthesia & 250 & NA & NA & -22.8 \\
\hline
\end{tabular}

Note:-L indicates left; R, right; SWI $\downarrow$, hypointensity; MRA $\downarrow$, decreased arterial caliber in the MCA territory; MRA $\uparrow$, increased arterial caliber in the MCA territory.

${ }^{a}$ Additional decrease in caliber of the posterior cerebral artery.

${ }^{\mathrm{b}}$ Additional decrease in caliber of the posterior and anterior cerebral arteries.

hours after symptom onset. Pollock et al ${ }^{14}$ similarly looked at a case series of 3 adults with migraine and noted cerebral hyperperfusion on ASL imaging $>6$ hours after the onset of neurologic symptoms.

Our results demonstrate a clear link between brain perfusion changes and the time course of migraine with aura. We observed a wide variation in the duration of perfusion changes, lasting up to at least 11 hours, the longest time to scan from symptom onset in this case series. Three patients in this series demonstrated increased perfusion on ASL within this time window, indicating the transition to hyperperfusion by $11,5.5$, and 7.5 hours. This is earlier than described in other studies. ${ }^{11-13}$ Each technique reviewed in this study provided data supporting the following pattern: apparent constriction of distal vessels on MRA, the presence of deoxygenated blood in cerebral veins on SWI, and initial hypoperfusion followed by a rebound hyperperfusion on ASL in the affected hemisphere. Patient 10 in this series demonstrated normal SWI and MRA findings and an ASL sequence with rCBF of only -0.4 at 4 hours, which may represent near-resolution of MR imaging perfusion changes or the transition from hypoperfusion to pseudonormalization during this patient's migraine. Most interesting, patient 7 in this series demonstrated discordance between the MR imaging sequences with a normal SWI signal and decreased vessel caliber on MRA but increased rCBF on pseudocontinuous ASL 5.5 hours from symptom onset. The discordance between pseudocontinuous ASL and SWI could indicate that ASL is more sensitive than SWI sequences in detecting imaging changes related to hemiplegic migraine. It could also indicate that the transition from hypoperfusion to hyperperfusion may be occurring sooner in the progression of migraine than previously thought, as observed in other case reports. ${ }^{14}$ The decreased vessel caliber on MRA with increased rCBF on ASL highlights the variability in neurovascular coupling and recovery characteristics with migraine. Migraine leads to reversible disturbances in neurovascular coupling that will affect the timing of ASL hyperperfusion and normalization of perfusion. ${ }^{16}$

Current models of migraine suggest that cortical spreading depression is a likely cause of the migraine aura. Spreading depression is a neurovascular phenomenon in which a self-propagating wave of depolarization results in spreading cortical hypoactivity and resultant vasoconstriction. Our data support this model through 2 observations: First, the distribution of changes across all sequences (ASL, SWI, MRA) cross vascular territories but respect the hemispheric boundary. Second, ASL findings demonstrate a trend of initial hemispheric hypoperfusion followed by remote rebound hyperperfusion hours later. In our imaging studies, the spatial resolution of ASL was not sufficient to allow more detailed analysis correlating symptoms and spreading depression localization. ${ }^{5}$ The decreased caliber in the posterior and/or anterior circulation observed in 4 of 12 patients in the present study confirms that multiple vascular territories are affected during migraine with aura. ${ }^{16}$

One limitation of this series is the lack of standardized documentation detailing the time course of each patient's clinical symptoms, including aura/headache onset and duration. This may explain some of the variability in rCBF findings. This discordance also emphasizes the value of obtaining multiple imaging sequences to assist in interpreting the patient's symptoms. An additional limitation of this study is that brain MR imaging was performed at only 1 time point rather than $\geq 2$ time points during the brain attack. Multiple samples of imaging data would allow more effective characterization of the evolution of perfusion changes during the migraine attack. Another limitation of this study is that for many of these patients, this was the first or only known attack of migraine with focal neurologic symptoms, which would not fulfill the International Classification of Headache Disorders, 3rd edition, criteria for migraine with aura or hemiplegic migraine. ${ }^{2}$

The management and disposition of migraine with aura differs remarkably from the entities in the differential diagnosis. Given the history and imaging findings, more than half of the patients in the series were not admitted to the hospital for a work-up and monitoring as would be typical for TIA; they were, instead, referred to the outpatient neurology clinic to establish headache care. Most patients were offered migraine prophylaxis treatment options (daily magnesium, riboflavin, tricyclic antidepressant, or antiepileptic). Given this difference, continuing to define criteria (both clinical and imaging) to identify and separate migraine from ischemic stroke in children by implementing noninvasive neuroimaging protocols in the emergency departments would be highly beneficial. This case series supports the addition of an ASL 
sequence in the evaluation of pediatric patients presenting with an acute brain attack.

\section{CONCLUSIONS}

The systematic use of multimodal MR imaging sequences including ASL, SWI, MRA, and DWI allows practitioners to distinguish hemiplegic migraine from stroke, with ASL being the most sensitive sequence to detect hemiplegic migraine. Our data suggest that the phenomenon of rebound hyperperfusion occurs earlier during a migraine attack than has been previously reported.

\section{REFERENCES}

1. Mackay MT, Chua ZK, Lee M, et al. Stroke and nonstroke brain attacks in children. Neurology 2014;82:1434-40 CrossRef Medline

2. Headache Classification Committee of the International Headache Society (IHS). The International Classification of Headache Disorders, 3rd edition. Cephalalgia 2018;38:1-211 CrossRef Medline

3. Hadjikhani N, Sanchez Del Rio M, Wu O, et al. Mechanisms of migraine aura revealed by functional MRI in human visual cortex. Proc Natl Acad Sci U S A 2001;98:4687-92 CrossRef Medline

4. Pietrobon D. Lessons from familial hemiplegic migraine and cortical spreading depression. In: Dalkara T, Moskowitz MA, eds. Neurobiological Basis of Migraine. Hoboken: John Wiley \& Sons; 2017:251-52

5. Grade M, Hernandez Tamames JA, Pizzini FB, et al. A neuroradiologist's guide to arterial spin labeling MRI in clinical practice. Neuroradiology 2015;57:1181-202 CrossRef Medline

6. Altinok D, Agarwal A, Ascadi G, et al. Pediatric hemiplegic migraine: susceptibility weighted and MR perfusion imaging abnormality. Pediatr Radiol 2010;40:1958-61 CrossRef Medline
7. Bosemani T, Burton VJ, Felling RJ, et al. Pediatric hemiplegic migraine: role of multiple MRI techniques in evaluation of reversible hypoperfusion. Cephalalgia 2014;34:311-15 CrossRef Medline

8. Fedak EM, Zumberge NA, Heyer GL. The diagnostic role for susceptibility-weighted MRI during sporadic hemiplegic migraine. Cephalalgia 2013;33:1258-63 CrossRef Medline

9. Gocmen R, Gunbey C, Arsava EM, et al. Susceptibility-weighted magnetic resonance imaging findings of two pediatric migraine patients with aura. Neuropediatrics 2016;47:46-50 CrossRef Medline

10. Chen J, Licht DJ, Smith SE, et al. Arterial spin labeling perfusion MRI in pediatric arterial ischemic stroke: initial experiences. $J$ Magn Reson Imaging 2009;29:282-90 CrossRef Medline

11. Boulouis G, Shotar E, Dangouloff-Ros V, et al. Magnetic resonance imaging arterial-spin-labeling perfusion alteration in childhood migraine with atypical aura: a case-control study. Dev Med Child Neurol 2016;58:965-69 CrossRef Medline

12. Lehman LL, Danehy AR, Trenor CC 3rd, et al. Transient focal neurologic symptoms correspond to regional cerebral hypoperfusion by MRI: a stroke mimic in children. AJNR Am J Neuroradiol 2017; 38:2199-202 CrossRef Medline

13. Iizaku T, Tominaga N, Kaneko J, et al. Biphasic neurovascular changes in prolonged migraine aura in familial hemiplegic migraine type 2. J Neurol Neurosurg Psychiatry 2015;86:344-53 CrossRef Medline

14. Pollock JM, Deibler AR, Burdette JH, et al. Migraine associated cerebral hyperperfusion with arterial spin-labeled MR imaging. AJNR Am J Neuroradiol 2008;29:1494-97 CrossRef Medline

15. Barber PA, Demchuk AM, Zhang J, et al; ASPECTS Study Group. Validity and reliability of a quantitative computed tomography score in predicting outcome of hyperacute stroke before thrombolytic therapy. Lancet 2000;355:1670-74 CrossRef Medline

16. Wolf ME, Held VE, Förster A, et al. Pearls \& oy-sters: dynamics of altered cerebral perfusion and neurovascular coupling in migraine aura. Neurology 2011;77:e127-28 CrossRef Medline 